\title{
Multifocal Electroretinogram in Hydroxychloroquine-related Macular Toxicity
}

\author{
Parveen Sen, Priya Ravi, Rupak Roy \\ Shri Bhagwan Mahavir Vitreoretinal Services, Sankara Nethralaya, Medical Research \\ Foundation, Chennai, India
}

\begin{abstract}
Prolonged use of hydroxychloroquine is common in patients with rheumatoid arthritis. Retinal toxicity due to hydroxychloroquine is known, and is monitored by investigations such as visual acuity, Amsler chart, colour vision, automated perimetry, full-field electroretinogram, and electrooculogram. Multifocal electroretinogram can suggest macular toxicity due to hydroxychloroquine even when the other investigations have normal results. Hence, multifocal electroretinogram has a role in the screening and follow-up of patients with suspected hydroxychloroquine toxicity.
\end{abstract}

Key words: Drug toxicity, Electroretinography, Hydroxychloroquine

Asian J Ophthalmol. 2011;12:224-8.

\section{Introduction}

Antimalarial agents are often used for a prolonged period in patients with rheumatoid arthritis. Hydroxychloroquine $(\mathrm{HQ})$ is less toxic than chloroquine and some other disease-modifying drugs. Common systemic side effects of $\mathrm{HQ}$ include gastrointestinal upset, skin rash, and headache. ${ }^{1}$ Keratopathy in the form of corneal deposits, lens opacities, and maculopathy causing a bull's eye lesion are the known ocular side effects of prolonged use of $\mathrm{HQ}{ }^{2}{ }^{2}$

Retinal toxicity with prolonged chloroquine and $\mathrm{HQ}$ use is due to primary retinal photoreceptor damage or damage to the retinal pigment epithelium. These drug effects are irreversible and progressive, even after cessation of the drug. ${ }^{3}$ Therefore, timely intervention is needed to avoid any visual handicap.

Diagnosis of retinal toxicity poses a challenge to ophthalmologists, and is difficult to diagnose in the early stages as the drug effects may be localised and not easily picked up by conventional electro-diagnostic procedures, which rely on the mass response of the retina. Investigations such as visual acuity, Amsler chart, colour vision, visual field analysis, and fundus examination are used to determine retinal toxicity in patients with a history of $\mathrm{HQ}$ intake. A paracentral scotoma on visual field testing may be the first indication of retinal toxicity secondary to $\mathrm{HQ} .^{4}$

There is a need for investigations that can document the focal toxic effects of HQ. This report highlights the role of multifocal

Correspondence: Dr Parveen Sen, Shri Bhagwan Mahavir Vitreoretinal Services, Sankara Nethralaya, Medical Research Foundation,

18 College Road, Chennai 600006, India.

Tel: (91 44) 2827 1616;

E-mail: parveensen@gmail.com electroretinogram (mfERG) in evaluating retinal toxicity in a patient with prolonged $\mathrm{HQ}$ intake.

\section{Case Report}

A 20-year-old presented in 2007 with a history of gradual painless decrease in vision for 2 years. She had been taking $\mathrm{HQ}$ for rheumatoid arthritis for the previous 3 years at doses of $400 \mathrm{mg}$ daily for the first year followed by $200 \mathrm{mg}$ daily for the next 2 years. Given that her weight was $54 \mathrm{~kg}$, her dose of $\mathrm{HQ}$ was $7.5 \mathrm{mg} / \mathrm{kg} / \mathrm{day}$ in the first year and $3.7 \mathrm{mg} / \mathrm{kg} /$ day in the next 2 years. The cumulative dose was $146 \mathrm{~g}$ in the first year and $73 \mathrm{~g}$ per year for the next 2 years, for a total of $292 \mathrm{~g}$ in 3 years.

Her best-corrected visual acuity (BCVA) was 6/18 in both eyes. Her BCVA recorded 4 years previously was $6 / 6$. Anterior segment examination and intraocular pressure were normal. No subepithelial corneal deposits were seen. Colour vision by Ishihara plates and Amsler chart were normal. Fundus examination revealed a few faint yellowish lesions at the level of the retinal pigment epithelium (RPE) in the macula in both eyes. Fundus fluorescein angiography showed window defects corresponding to the RPE lesions (Figure 1). Visual field testing with the Swedish Interactive Thres-holding Algorithm fast Humphrey field analyser 30-2 revealed a few paracentral defects. Given the patient's drug history, a diagnosis of retinal toxicity following $\mathrm{HQ}$ use was considered.

Full-field ERG (ffERG) and mfERG were recorded using the VERIS ${ }^{\text {TM }}$ Science 5.1 system (EDI, Inc, San Mateo, USA) following the guidelines of the International Society for Clinical Electrophysiology of Vision. ${ }^{5,6}$ The stimulus for mfERG consisted of 103 hexagons 
Figure 1. Fundus photographs of the right and the left eyes showing (a) yellowish lesions at the level of the retinal pigment epithelium; and (b) corresponding window defects seen on fundus fluorescein angiography.
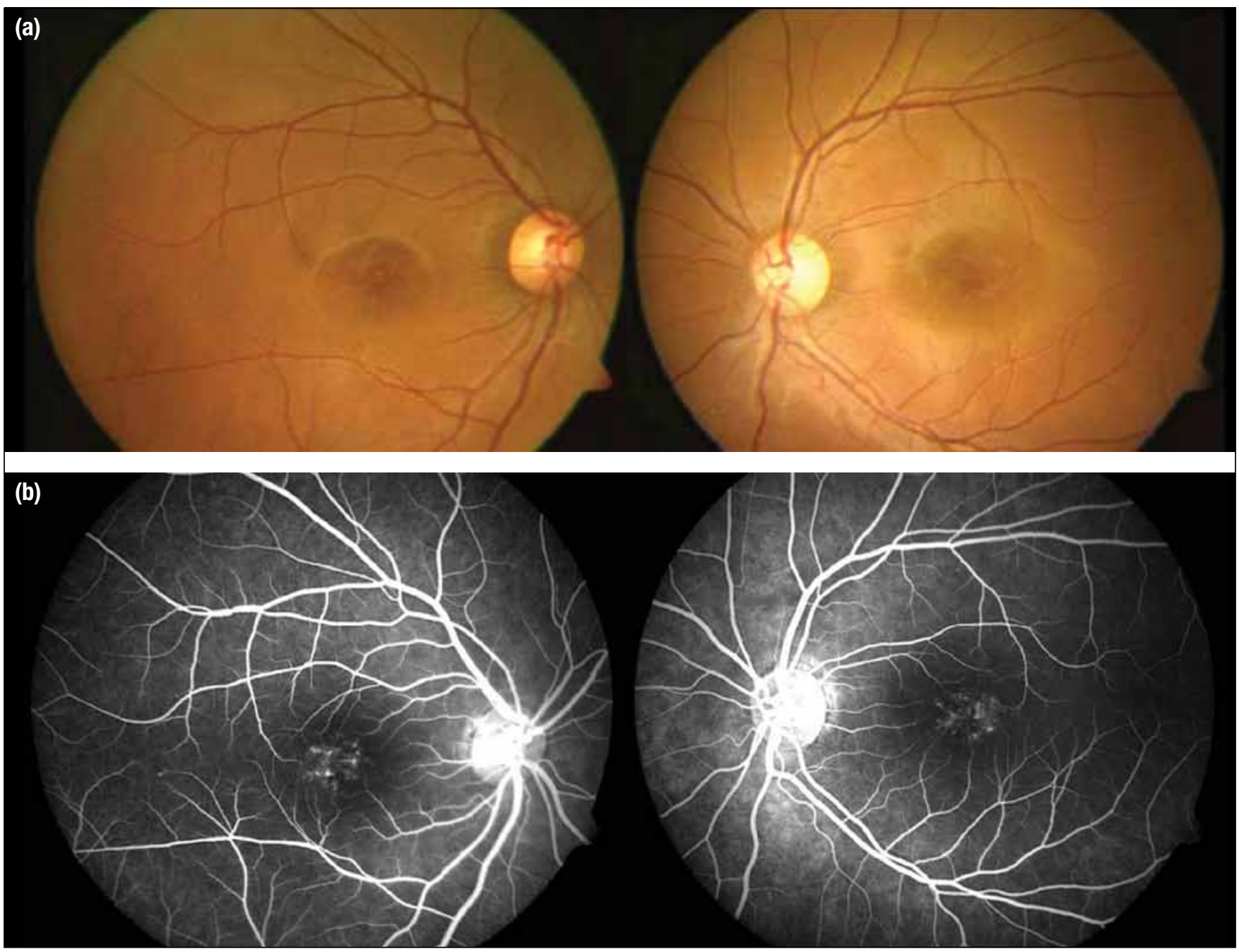

viewed at a distance of $53 \mathrm{~cm}$ and subtended $35^{\circ}$ horizontally and $31^{\circ}$ vertically in the visual field. The 103 hexagons appeared to flicker according to a pseudorandom binary $m$-sequence of black and white hexagonal presentations. A Grass 15LT amplifier (Astro-Med, Inc, West Warwick, USA) with band pass from 10 to $300 \mathrm{~Hz}$ and gain of 50,000 was used. The selected pseudorandom binary m-sequence was $2^{15-1}$, the stimulation rate was $75 \mathrm{~Hz}$ (interframe base interval, $13.33 \mathrm{~ms}$ ), and the response signal was sampled at 16 times per frame (interval, $0.83 \mathrm{~ms}$ ), resulting in a recording time of approximately 7 minutes 17 seconds. The raw waveform was visible throughout the recording and segments were rejected if there were any artefacts due to excessive blinking or poor fixation. ${ }^{6}$ Ambient room lighting was used during mfERG recording. The stimulus pattern comprised a central hexagon corresponding to the fovea and the five concentric rings at different eccentricities corresponding to the paramacular region. Ring 1 (R1) subtended $<1.6^{\circ}$ in diameter, ring 2 (R2) was $1.6^{\circ}$ to $6.0^{\circ}$, ring $3(\mathrm{R} 3)$ was $6.0^{\circ}$ to $11.4^{\circ}$, ring 4 (R4) was $11.4^{\circ}$ to $18.2^{\circ}$, ring 5 (R5) was $18.2^{\circ}$ to $26.2^{\circ}$, and ring 6 (R6) was $26.2^{\circ}$ to $35.0^{\circ}$.

Both ffERG and mfERG recordings were done using Burien-Allen bipolar contact lens electrodes (Hansen Ophthalmic Laboratories, lowa City, USA) with the ground electrode placed on the earlobe. Parameters measured were amplitudes and implicit times. The amplitudes were measured in $\mu \mathrm{V}$ for ffERG and $\mathrm{nV} / \mathrm{deg}^{2}$ for mfERG, and implicit times were measured in ms.

The first order kernels of mfERG (N1 and P1 implicit times, amplitudes) were taken for analysis. The mean N1, P1 implicit times and amplitudes for each ring from the mfERG responses of both eyes were calculated and compared with those of age-matched controls $(n=13)$.

ffERG showed normal scotopic and photopic responses (Figure 2), but mfERG revealed a significant decrease in amplitudes and increase in implicit times of $\mathrm{N} 1$ and $\mathrm{P} 1$ in rings 1 and 2 (Figure 3).The 
Figure 2. Full-field electroretinograms of the right and left eyes showing normal scotopic and photopic responses.

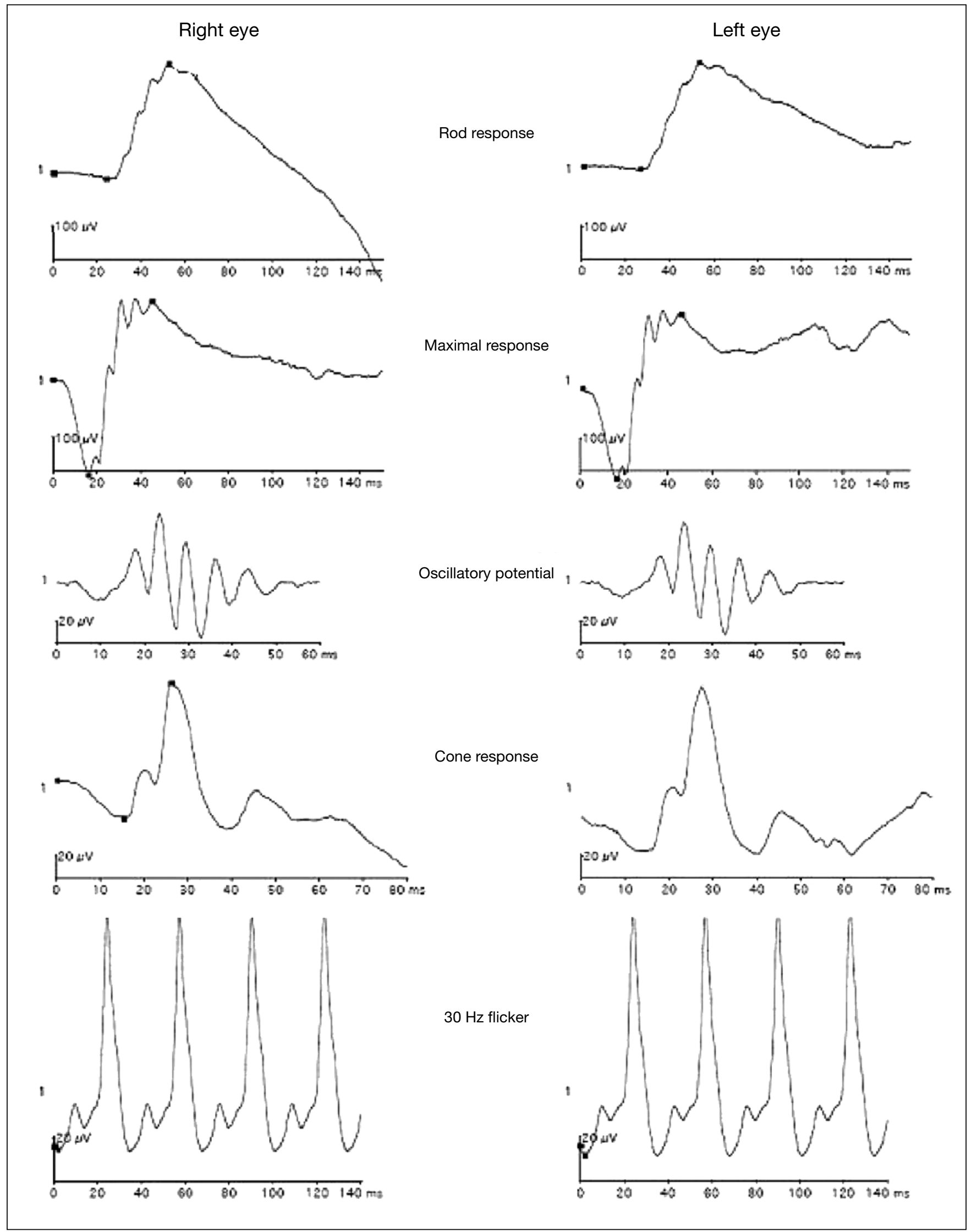


Figure 3. Multifocal electroretinogram trace array and 3-dimensional chart showing a significant decrease in the central ring responses.

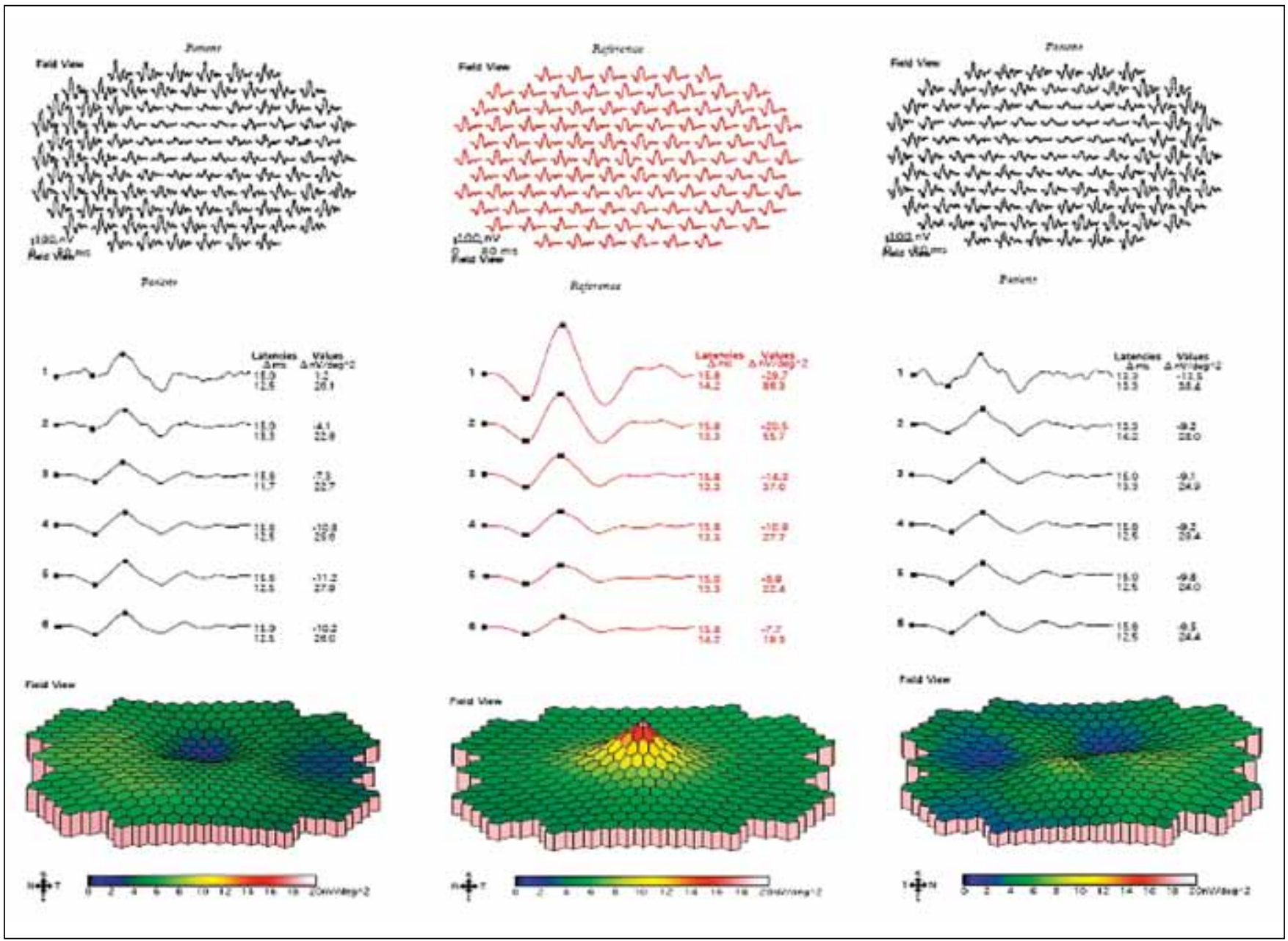

responses in the peripheral rings were comparable with those of age-matched controls (Figure 4).

\section{Discussion}

Retinal toxicity due to $\mathrm{HQ}$ has decreased as the dose of $\mathrm{HQ}$ prescribed for arthritis has been optimised over the years. Sightthreatening retinopathy in these patients occurs less frequently at the currently recommended dose of $400 \mathrm{mg}$ daily. ${ }^{2}$ In 2002, the American Academy of Ophthalmology recommended that patients taking $\mathrm{HQ}$ with normal baseline investigations do not require special ophthalmological monitoring for 5 years provided that the daily dose of $\mathrm{HQ}$ is less than $6.5 \mathrm{mg} / \mathrm{kg}$ body weight and the cumulative dose does not exceed $200 \mathrm{~g} .{ }^{7}$ However, retinopathy has been noticed at drug doses that are considered 'safe' ( $<6.5 \mathrm{mg} / \mathrm{kg}$ body weight). ${ }^{8}$ For this patient, the cumulative dose was more than $200 \mathrm{~g}$ and the dose per year was more than the recommended dose. The patient did not have any high risk factors such as kidney or liver disease or high body fat. ${ }^{7}$ Although she had experienced a decrease in vision over 2 years, she had no history of prior screening for $\mathrm{HQ}$ toxicity.
Therefore, awareness of the treating physician is needed to optimise the drug dose while considering the risks and benefits of $\mathrm{HQ}$. Asian patients, especially women, often weigh less than western women, and this must be considered when adjusting the dose.

Various screening tests have been proposed to detect retinal lesions due to $\mathrm{HQ}$, including visual acuity testing, automated perimetry, Amsler chart, colour vision, indirect ophthalmoscopy, ERG, and electrooculogram. For this patient, fundus examination, colour vision, and Amsler chart did not reveal the typical features of bull's eye maculopathy, and ffERG was non-committal. However, mfERG recordings revealed a decrease in central retinal responses, as seen by Maturi et al ${ }^{9}$ and Lai et al. ${ }^{10}$ The pattern of a central depression with normal peripheral responses is one of the typical presentations of $\mathrm{HQ}$ toxicity. Automated perimetry also showed paracentral defects. However, perimetry is a subjective evaluation, while $\mathrm{mfERG}$ is an objective technique for detecting the topography of the toxicity, ${ }^{11,12}$

Although mfERG is a useful method for early detection of retinal toxicity, its application may be limited because of inadequate 
Figure 4. Multifocal electroretinogram graphs depicting (a) N1 amplitude; (b) P1 amplitude; (c) N1 implicit times; and (d) P1 implicit times compared with agematched controls.

(a)

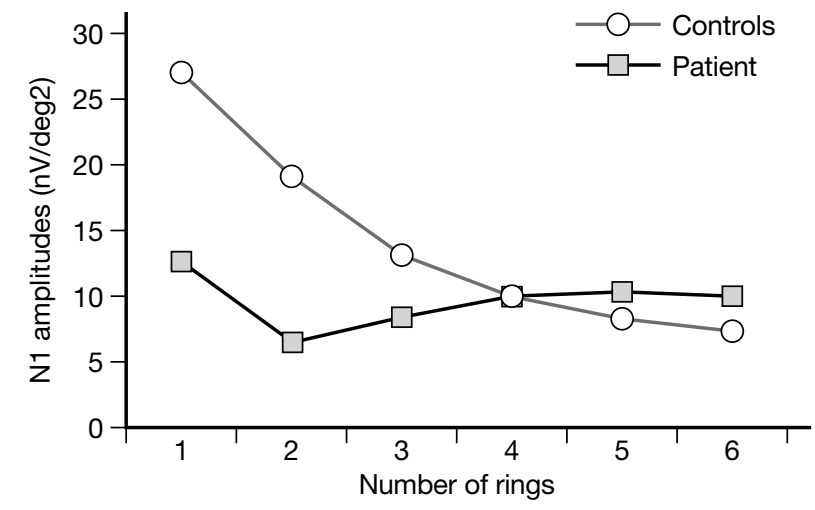

(c)

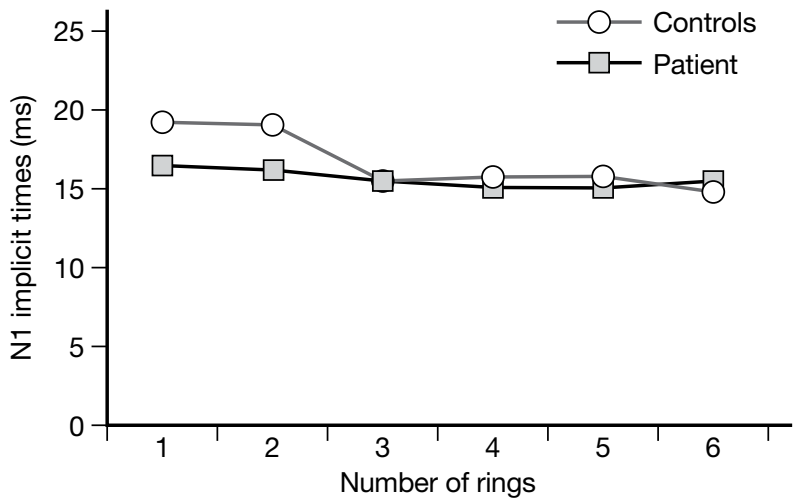

(b)

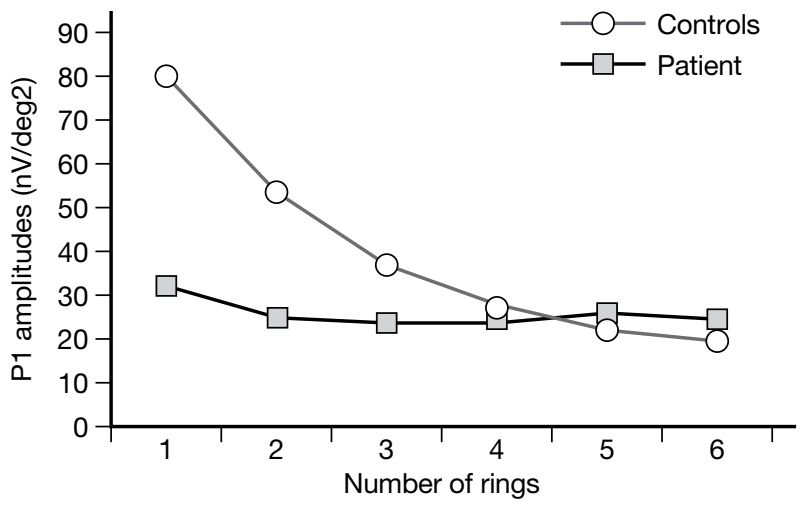

(d)

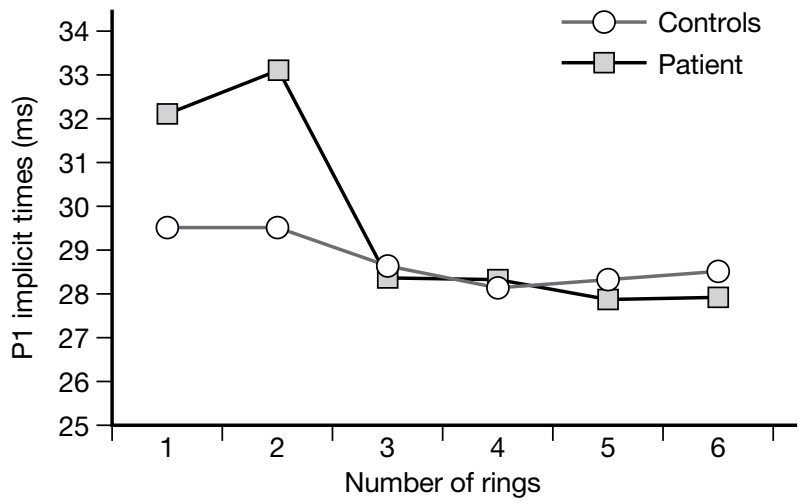

access to this technology in many ophthalmic centres, especially in developing nations in Asia. Incorporation of an appropriate ophthalmic screening programme for the management of patients with $\mathrm{HQ}$ toxicity needs to be considered to prevent permanent visual loss. Base-line ffERG should be done before administering $H Q$. ffERG and mfERG compliment each other, and mfERG can detect macular toxicity even when other investigations are normal.

\section{References}

1. van Jaarsveld $\mathrm{CH}$, Jahangier $\mathrm{ZN}$, Jacobs JW, et al. Toxicity of antirheumatic drugs in a randomized clinical trial of early rheumatoid arthritis. Rheumatology (Oxford). 2000;39:1374-82.

2. Bernstein $\mathrm{HN}$. Ophthalmologic considerations and testing in patients receiving long-term antimalarial therapy. Am J Med. 1983;75:25-34.

3. Sassani JW, Brucker AJ, Cobbs W, Campbell C. Progressive chloroquine retinopathy. Ann Ophthalmol. 1983;15:19-22.

4. Yam JC, Kwok AK. Ocular toxicity of hydroxychloroquine. Hong Kong Med J. 2006;12:294-304.

5. Marmor MF, Fulton AB, Holder GE, Miyake Y, Brigell M, Bach M;
International Society for Clinical Electrophysiology of Vision. ISCEV Standard for full-field clinical electroretinography (2008 update). Doc Ophthalmol. 2009;118:69-77.

6. Hood DC. Assessing retinal function with the multifocal technique. Prog Retin Eye Res. 2000;19: 607-46.

7. Marmor MF, Carr RE, Easterbrook M, Farjo AA, Mieler WF. Recommendations on screening for chloroquine and hydroxychloroquine retinopathy: a report by the American Academy of Ophthalmology. Ophthalmology. 2002;109:1377-82.

8. Bienfang D, Coblyn JS, Liang MG, Corzillius M. Hydroxychloroquine retinopathy despite regular ophthalmologic evaluation: a consecutive series. J Rheumatol. 2000;27:2703-6.

9. Maturi RK, Yu M, Weleber RG. Multifocal electroretinographic evaluation of long-term hydroxychloroquine users. Arch Ophthalmol. 2004;122:973-81.

10. Lai TY, Chan WM, Li H, Lai RY, Lam DS. Multifocal electroretinographic changes in patients receiving hydroxychloroquine therapy. Am J Ophthalmol. 2005;140:794-807.

11. Marmor MF. The dilemma of hydroxychloroquine screening: new information from the multifocal ERG. Am J Ophthalmol. 2005;140: 894-5.

12. Gilbert ME, Savino PJ. Missing the bull's eye. Surv Ophthalmol. 2007;52:440-2. 